\title{
PLANE NETS PERIODIC OF PERIOD 3 UNDER THE LAPLACIAN TRANSFORMATION.
}

\author{
By Jasper Ole Hassler (Chicago, U.S. A.).
}

Adunanza del 14 novembre 1915.

INTRODUCTION.

The analytic basis for the projective theory of a net of plane curves as developed by Professor WiLczYnski ${ }^{2}$ ) is briefly as follows:

Let the homogeneous coordinates of a point in a plane be given as functions of two independent variables, $u$ and $v$, so that

$$
y^{(k)}=f^{(k)}(u, v)
$$$$
(k=\mathrm{I}, 2,3) \text {. }
$$

The equations $u=$ const. and $v=$ const. will then in general give rise to two oneparameter families of plane curves which together constitute the net under consideration. This net will degenerate if and only if the determinant

vanishes identically.

$$
D=\left|\begin{array}{ccc}
y_{u}^{(1)} & y_{u}^{(2)} & y_{u}^{(3)} \\
y_{v}^{(1)} & y_{v}^{(2)} & y_{v}^{(3)} \\
y^{(1)} & y^{(2)} & y^{(3)}
\end{array}\right|
$$

Every parametric representation of form (I) for a non-degenerate net of plane curves determines a unique system of partial differential equations of the form

$$
\left\{\begin{array}{l}
y_{u u}=a y_{u}+b y_{v}+c y, \\
y_{u v}=a^{\prime} y_{u}+b^{\prime} y_{v}+c^{\prime} y, \\
y_{v v}=a^{\prime \prime} y_{u}+b^{\prime \prime} y_{v}+c^{\prime \prime} y,
\end{array}\right.
$$

of which $y^{(1)}, y^{(2)}, y^{(3)}$ are a fundamental system of solutions and whose integrability conditions are satisfied on account of the assumptions made on $y^{(1)}, y^{(2)}, y^{(3)}$.

Conversely, the members $y^{(1)}, y^{(2)}, y^{(3)}$ of any fundamental system of solutions of a completely integrable system of form (2) may be interpreted as the homogeneous coordinates of a point in a plane, thus defining a non-degenerate plane net. The net

I) E. J. Wilczynski, One-Parameter Families and Nets of Plane Curves [Transactions of the American Mathematical Society, vol. XII (1911), pp. 473-510]. 
defined by any other fundamental system of (2) will then be a projective transformation of this first one.

The system of partial differential equations (2) may be transformed into a canonical form whose coefficients depend only upon the rations of the homogeneous coordinates $y^{(1)}, y^{(2)}, y^{(j)}$ of the net and not upon their absolute values. This canonical form may be obtained as follows: If we make the transformation

(3)

where

$$
y=\lambda(u, v) \bar{y},
$$

$$
\lambda=\text { const. } e^{\frac{1}{3} f}
$$

$f$ being defined by the equations

$$
a+b^{\prime}=f_{u}, \quad a^{\prime}+b^{\prime \prime}=f_{v},
$$

the resulting system is said to be in its canonical form. We find the following values for the coefficients of the canonical form ${ }^{2}$ ) of the system:

$$
\begin{aligned}
& A=a-\frac{2}{3} f_{u}, \quad B=b, \quad C=c+\frac{1}{3} a f_{u}+\frac{1}{3} b f_{v}-\frac{1}{3} f_{u u}-\frac{1}{9} f_{u}^{2}, \\
& A^{\prime}=a^{\prime}-\frac{1}{3} f_{v}, \quad B^{\prime}=b^{\prime}-\frac{1}{3} f_{u}, \quad C^{\prime}=c^{\prime}+\frac{1}{3} a^{\prime} f_{u}+\frac{1}{3} b^{\prime} f_{v}-\frac{1}{3} f_{u v}-\frac{1}{9} f_{u} f_{v}, \\
& A^{\prime \prime}=a^{\prime \prime}, \quad B^{\prime \prime}=b^{\prime \prime}-\frac{2}{3} f_{v}, \quad C^{\prime \prime}=c^{\prime \prime}+\frac{1}{3} a^{\prime \prime} f_{u}+\frac{1}{3} b^{\prime \prime} f_{v}-\frac{1}{3} f_{v v}-\frac{1}{9} f_{v}^{2} \text {. }
\end{aligned}
$$

It is easy to verify that

$$
A+B^{\prime}=\text { o, } \quad A^{\prime}+B^{\prime \prime}=\text { o, }
$$

and these conditions are characteristic of the canonical form.

The coefficients $A, B, C, \ldots$ of a system in its canonical form are seminvariants of system (2). That is, their values are not changed by any transformation of the form $y=\lambda \bar{y}$ where $\lambda$ is an arbitrary function of $u$ and $v$. It is this fact which proves that $A, B, C$, are functions of the ratios $y^{(1)}: y^{(2)}: y^{(3)}$ only. The fundamental covariants ${ }^{3}$ ) of the system (2) are $y$ and

$$
\rho=y_{u}-b^{\prime} y, \quad \sigma=y_{v}-a^{\prime} y .
$$

The integrability conditions for a system in its canonical form are

$$
\left\{\begin{array}{l}
B_{v}^{\prime}+A_{u}^{\prime}+C^{\prime}+A^{\prime} B^{\prime}-A^{\prime \prime} B=0 \\
B_{v}-B_{u}^{\prime}=-C+2 B^{\prime 2}+2 A^{\prime} B \\
C_{v}-C_{u}^{\prime}=2 B^{\prime} C^{\prime}+A^{\prime} C-B C^{\prime \prime} \\
A_{v}^{\prime}-A_{u}^{\prime \prime}=C^{\prime \prime}-2 A^{\prime 2}-2 A^{\prime \prime} B^{\prime} \\
\left.C_{v}^{\prime}-C_{v}^{\prime \prime}=-2 A^{\prime} C^{\prime}+A^{\prime \prime} C-B^{\prime} C^{\prime \prime}{ }^{4}\right) .
\end{array}\right.
$$

The transformation of the form

$$
\bar{u}=U(u), \quad \bar{v}=V(v),
$$

where $U$ and $V$ are arbitrary functions of the single variables indicated, do not alter

2) loc. cit. ${ }^{\mathrm{I}}$ ), p. 476 .

3) loc. cit. ${ }^{\mathrm{I}}$ ), p. 477 .

${ }^{4)}$ loc. cit. ${ }^{\text {I}}$ ), p. 478 . 
the net but merely change its analytic representation. The following seven quantities ${ }^{5}$ )

$$
\left\{\begin{aligned}
\mathfrak{B} & =B, & \mathfrak{C} & =C-B_{u}^{\prime}-2 B^{\prime 2}+A^{\prime} B, \\
\mathfrak{A}^{\prime} & =A^{\prime}+\frac{\mathrm{I}}{6} \frac{A_{v \prime}^{\prime \prime},}{A^{\prime \prime}}, & \mathfrak{B} & =B^{\prime}+\frac{\mathrm{I}}{6} \frac{B_{u}}{B}, \quad \mathfrak{S}^{\prime}=C^{\prime}+A^{\prime} B^{\prime}, \\
\mathfrak{A}^{\prime \prime} & =A^{\prime \prime}, & \mathfrak{C}^{\prime \prime} & =C^{\prime \prime}-A_{v}^{\prime}-2 A^{\prime 2}+A^{\prime \prime} B^{\prime},
\end{aligned}\right.
$$

are left invatiant (except for a factor) by all transformations of the form (6). Moreover these seven functions determine the net completely, except for projective transformations, and are therefore called the fundamental invariants of the net. All other invariants of system (2) are functions of these and of their partial derivatives.

The integrability conditions (5) may be written in the following form ${ }^{6}$ )

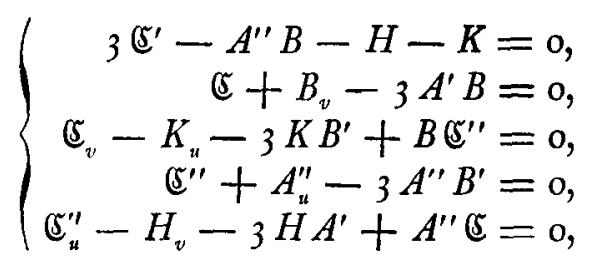

in terms of the invariants $(7)$ and the two further invariants

$$
H=C^{\prime}+A^{\prime} B^{\prime}-A_{u}^{\prime}, \quad K=C^{\prime}+A^{\prime} B^{\prime}-B_{v}^{\prime},
$$

which, although not independent of the seven invariants (7), are of special importance. In fact they are the only ones which had received systematic attention before the appearance of Professor WiLczynski's paper, being essentially identical with the invariants $b$ and $k$ of the equations of the form

$$
\frac{\partial^{2} z}{\partial x \partial y}+a \frac{\partial z}{\partial x}+b \frac{\partial z}{\partial y}+c z=0 \text {. }
$$

An extensive theory of these equations was begun by LAPLACE and built up by Darboux ${ }^{7}$ ). The geometric interpretations used by Darboux however were all connected with the theory of conjugate systems of curves on curved surfaces and not at all with the theory of plane nets.

For given values $u_{\mathrm{o}}$ and $v_{\mathrm{o}}$ of $u$ and $v$ the quantities $y^{(1)}, y^{(2)}, y^{(3)}$, defined by equations ( $I$ ) are the homogeneous coordinates of a point $P$ through which pass two curves of the net $u=u_{0}$ and $v=v_{0}$, respectively. If we substitute successively the three values of $y$ in (4) we get three corresponding values $p_{1}, p_{2}, p_{3}$ of $p$ which define a point $P_{p}$ of the line tangent to the curve $v=v_{0}$ at $P_{y}$. In a similar manner the point $P_{\sigma}$ is defined on the line tangent to the curve $u=u_{\mathrm{o}}$ at $P_{y}$. As $u$ and $v$ vary separately the point $P_{\sigma}$ describes certain curves, thus giving rise to a new net

5) loc. cit. ${ }^{1}$ ), equations (21).

6) loc. cit. ${ }^{2}$ ), equations (34).

7) G. Darboux, Leçons sur la theorie générale des surfaces et les applications géometriques $d u$ Calcul infinitésimal, $\mathrm{II}^{\mathrm{e}}$ Partie (Paris, Gauthier-Villars, 1889). 
called the first LapLacian transform of the original net. Similarly the net determined by $P_{\rho}$ is called the minus first Laplacian transform.

The tangent to the curve $u=$ const. of the original net at $P_{y}$ is at the same time tangent to the curve $v=$ const. of the first Laplacian transform at $P_{a}$. Similarly, the tangent to the curve $v=$ const. of the original net at $P_{y}$ is tangent to the curve $u=$ const. of the minus first Laplacian transform at $P_{\rho}$. The first Laplacian transform of the $\rho$-net and the minus first Laplacian transform of the $\sigma$-net both coincide with the original $y$-net. We shall not write down the systems of partial differential equations for the LapLAcian transforms except for the particular case which interests us in this paper ${ }^{8}$ ).

Let us use the triangle $P_{y} P_{p} P_{\sigma}$ as a local triangle of reference. More specifically, we introduce a local system of homogeneous coordinates such that any expression of the form

$$
x_{1} y^{(k)}+x_{2} \rho^{(k)}+x_{3} \sigma^{(k)} \quad(k=1,2,3),
$$

will represent a point whose coordinates referred to the local coordinate system are proportional to $x_{1}, x_{2}, x_{3}$, respectively. If we use this coordinate system the equations of the conics which osculate the curves $v=$ const. and $u=$ const., respectively, at the point $P_{y}$ have the form ${ }^{9}$ )

$$
\left\{\begin{array}{l}
M=\mathfrak{B}^{2} x_{2}^{2}+4 \mathfrak{B P}^{\prime} \mathfrak{B}_{2} x_{3}-2 \mathfrak{B} x_{1} x_{3}+\varphi x_{3}^{2}=0 \\
N=\psi x_{2}^{2}+4 \mathfrak{U}^{\prime} \mathfrak{U}^{\prime \prime} x_{2} x_{3}+\mathfrak{U}^{\prime \prime 2} x_{3}^{2}-2 \mathfrak{I}^{\prime \prime} x_{1} x_{2}=0
\end{array}\right.
$$

respectively, where

$$
\left\{\begin{array}{l}
\varphi=\mathfrak{C}-4 \mathfrak{B}^{\prime 2}+2 \mathfrak{B}_{u}^{\prime}+6 \mathfrak{B}^{\prime} B^{\prime}, \\
\psi=\mathfrak{C}-4 \mathfrak{H}^{\prime 2}+2 \mathfrak{A}_{v}^{\prime}+6 \mathfrak{A}^{\prime} A^{\prime},
\end{array}\right.
$$

In this paper we consider a certain class of nets, characterized by the invariantive conditions $\mathfrak{C}=\mathfrak{C}^{\prime \prime}=0$. In general these nets are periodic of period 3 under the LAPLACIan transformation. We shall study the seminvariants and invariants of such nets and their osculating conics both for the original net and for those obtained from it by the LAPLACIan transformation. We shall show just what the geometric elements are which determine a net of this kind giving rise to an existence theorem of a very interesting type.

Finally, we shall study more in detail a special case in which all of the curves of the net are conics and where the additional conditions $H=\mathrm{K}=\mathrm{o}$ are imposed.

The author takes this opportunity of expressing his indebtedness to Professor WiLczynski for the inspiration which led to this subject and for the advice so generously given during the preparation of this paper.

${ }^{8}$ ) For the general formulx see loc. cit. ${ }^{1}$ ), $\$ 4$.

9) loc. cit. ${ }^{\mathrm{I}}$ ), pp. 503, 504. 


$$
\int 1 \text {. }
$$

\section{The system of partial differential equations and the invariants for nets periodic of period 3 under the Laplacian transformation.}

If the first Laplacian transform of the $\sigma$-net be the $p$-net, and if the minus first transform of the $\rho$-net be the $\sigma$-net we have a closed system of nets containing the original net, its first and minus first Laplacian transforms. Symbolically, if $L$ denotes the LapLAcian transformation, we should then have

$$
\begin{aligned}
& L(y)=\sigma, \quad L^{2}(y)=L(\sigma)=\rho, \quad L^{3}(y)=L(\rho)=y, \\
& L^{-1}(y)=p, \quad L^{-2}(y)=L^{-1}(p)=\sigma, \quad L^{-j}(y)=L^{-1}(\sigma)=y \text {. }
\end{aligned}
$$

A net which satisfies these conditions would be periodic of period 3 under the LAPLAcian transformation.

The necessary and sufficient conditions for a net to be periodic of period 3 under the LAPLACIan transformation are

$$
\begin{aligned}
& \mathfrak{E}=\mathrm{o}, \quad \mathbb{E}^{\prime \prime}=\mathrm{o}, \\
& H \neq \mathrm{o}, \quad K \neq \mathrm{o} .
\end{aligned}
$$

To prove this we compute the covariants of the first and minus first Laplacian transforms. The covariant of the $\sigma$-net which corresponds to the covariant $\sigma$ of the $y$-net is

$$
\sigma_{1}=\sigma_{v}-a_{1}^{\prime} \sigma .
$$

From the general formulæ ${ }^{\text {io }}$ ) we have

$$
a_{\mathrm{s}}^{\prime}=A^{\prime}+\frac{H_{v}}{H}, \quad \sigma_{v}=\mathfrak{\mho}^{\prime \prime} y+A^{\prime \prime} \rho-2 A^{\prime} \sigma,
$$

whence

Similarly we find

$$
\sigma_{1}=\mathfrak{C}^{\prime \prime} y-\left[\frac{H_{v}}{H}+3 A^{\prime}\right] \sigma+A^{\prime \prime} \mathrm{p} .
$$

$$
\rho_{-1}=\varsigma_{y}-\left[\frac{K_{u}}{K}+3 B^{\prime}\right] \rho+B \sigma .
$$

Thus the point $P_{\sigma_{1}}$ will be on the line joining $P_{\sigma}$ to $P_{\rho}$ if and only if $\mathbb{C}^{\prime \prime}=0$, and the point $P_{P_{-I}}$ will be on this line if and only if $\mathfrak{C}=0$. But if $\mathfrak{C}=\mathfrak{C}^{\prime \prime}=0$ we obtain from $\left(8_{3}\right)$ and $\left(8_{5}\right)$, provided $H \neq 0$ and $K \neq 0$,

$$
\frac{H_{v}}{H}+3 A^{\prime}=0, \quad \frac{K u}{K}+3 B^{\prime}=0,
$$

and consequently

$$
\sigma_{1}=A^{\prime \prime} \rho_{,} \quad \rho_{-1}=B \sigma,
$$

and these equations prove our theorem.

ro) loc. cit. I), equations (43) and (93). 
From equations ( 12$),\left(8_{2}\right),\left(8_{4}\right)$ we obtain the relations

$$
3 A^{\prime}=\frac{B_{v}}{B}, \quad 3 B^{\prime}=\frac{A_{u}^{\prime \prime}}{A^{\prime \prime}} \text {. }
$$

It follows then from equations (14) and (15) that

whence

$$
\frac{H_{v}}{H}+\frac{B_{v}}{B}=0, \quad \frac{K_{u}}{K}+\frac{A_{u}^{\prime \prime}}{A^{\prime \prime}}=\mathrm{o},
$$

$$
H B=\psi(u), \quad K A^{\prime \prime}=\psi(v),
$$

if $\varphi$ and $\psi$ are arbitrary functions of $u$ and $v$ alone, respectively.

The products $H B$ and $K A^{\prime \prime}$ are relative invariants. If we apply the transformation (6) they are transformed into

$$
\bar{H} \bar{B}=\frac{\mathrm{I}}{\left(U^{\prime}\right)^{3}} H B, \quad \bar{K} \overline{A^{\prime \prime}}=\frac{\mathrm{I}}{\left(V^{\prime}\right)^{3}} K A^{\prime \prime} .
$$

If, therefore, we choose $U$ and $V$ so that $\left(U^{\prime}\right)^{3}=\varphi(u)$ and $\left(V^{\prime}\right)^{3}=\psi(v)$ we have

$$
H B=\mathrm{I}, \quad K A^{\prime \prime}=\mathrm{I} \text {. }
$$

Since this transformation does not involve the geometric properties of the net, but merely determines in part a special form of its parametric representation, we may assume this transformation to have been made at the outset, so that we shall have

$$
H=\frac{\mathrm{I}}{B}, \quad K=\frac{\mathrm{I}}{A^{\prime \prime}} \text {. }
$$

We may now express all of the coefficients of the canonical form in terms of the two seminvariants $B$ and $A^{\prime \prime}$ (these quantities are also invariants). In fact, using the equations (7), (8), (12), (15), (16), we find
(17) $\left\{\begin{array}{l}A=-B^{\prime}, \\ A^{\prime}=\frac{B_{v}}{3 B}, \\ A^{\prime \prime}=A^{\prime \prime}\end{array}\right.$

$$
B=B \text {, }
$$$$
C=\frac{A_{u u}^{\prime \prime}}{3 A^{\prime \prime}}-\frac{A_{u}^{\prime \prime 2}}{9 A^{\prime \prime 2}}-\frac{B_{v}}{3},
$$$$
B^{\prime}=\frac{A_{u}^{\prime \prime}}{3 A^{\prime \prime}},
$$$$
C^{\prime}=\frac{\mathrm{I}}{3}\left(A^{\prime \prime} B+\frac{\mathrm{I}}{A^{\prime \prime}}+\frac{\mathrm{I}}{B}\right)-\frac{A_{u}^{\prime \prime} B_{v}}{9 A^{\prime \prime} B},
$$$$
B^{\prime \prime}=-A^{\prime} \text {, }
$$$$
C^{\prime \prime}=\frac{B_{v v}}{3 B}-\frac{B_{v}^{2}}{9 B^{2}}-\frac{A_{u}^{\prime \prime}}{3} \text {. }
$$

From equations (9) and (16) we obtain the two further conditions

$$
\left\{\begin{array}{l}
\frac{\partial^{2} \log A^{\prime \prime}}{\partial u \partial v}=A^{\prime \prime} B-\frac{2}{A^{\prime \prime}}+\frac{\mathrm{I}}{B}, \\
\frac{\partial^{2} \log B}{\partial u \partial v}=A^{\prime \prime} B+\frac{\mathrm{I}}{A^{\prime \prime}}-\frac{2}{B} .
\end{array}\right.
$$

On the other hand, if we consider a system of partial differential equations in the canonical form

$$
\left\{\begin{array}{l}
y_{u t}=-B^{\prime} y_{u}+B y_{v}+C y, \\
y_{u v}=A^{\prime} y_{u}+B^{\prime} y_{v}+C^{\prime} y, \\
y_{v v}=A^{\prime \prime} y_{u}-A^{\prime} y_{v}+C^{\prime \prime} y^{\prime},
\end{array}\right.
$$


whose coefficients satisfy the conditions ( 17 ) and (I 8 ) we find that the integrability conditions $(5)$ are identically satisfied and that $\mathfrak{s}=\mathfrak{S}^{\prime \prime}=0$. Hence we may formulate the following theorem:

If the coefficients of a system of partial differential equations of the form (19) satisfy conditions ( 17 ) and ( 18 ) and if $H$ and $K$ are different from zero, any fundamental system of solutions $y^{(3)}, y^{(2)}, y^{(3)}$ of (19) defines a net, periodic of period 3 under the LAPLACIan transformation, of which the most general net of period 3 is a projective transformation. Conversely, any non-degenerate net of period 3, defined by (I), whose first and minus first LAPLACian transforms are non-degenerate gives rise to a system of partial differential equations of the form (19) whose coefficients satisfy the conditions (17) and (18).

The invariants of the system defined by equations (17), (18) and (19) are given by

(20) $\left\{\begin{array}{rlrl}\mathfrak{B} & =B, & \mathfrak{E} & =\mathrm{o}, \\ \mathfrak{I}^{\prime} & =\frac{B_{v}}{3 B}+\frac{A_{v}^{\prime \prime}}{6 A^{\prime \prime}}, & \mathfrak{B}^{\prime} & =\frac{A_{u}^{\prime \prime}}{3 A^{\prime \prime}}+\frac{B_{u}}{6 B}, \quad \mathbb{S}^{\prime}=\frac{\mathrm{I}}{3}\left(A^{\prime \prime} B+\frac{\mathrm{I}}{A^{\prime \prime}}+\frac{\mathrm{I}}{B}\right), \\ \mathfrak{I}^{\prime \prime} & =A^{\prime \prime}, & \mathfrak{S}^{\prime \prime}=\mathrm{o}, \quad H=\frac{\mathrm{I}}{B}, \quad K=\frac{\mathrm{I}}{A^{\prime \prime}},\end{array}\right.$ and the covariants are $(20 a)$

$$
\rho=y_{u}-\frac{A_{u}^{\prime \prime}}{3 A^{\prime \prime}} y, \quad \sigma=y_{v}-\frac{B_{v}}{3 B} y .
$$

The seminvariants and invariants of the Laplacian transforms.

We shall now list ${ }^{17}$ ), for later use, the seminvariants of the first LAplacian transform. They have the form

(21) $\left\{\begin{array}{c}A_{t}^{\prime \prime}=-B_{\mathrm{t}}^{\prime}, \quad B_{\mathrm{t}}=\frac{\mathrm{I}}{A^{\prime \prime} B}, \\ C_{\mathrm{1}}=\frac{B_{u u}}{3 B}-\frac{\mathrm{I}}{9} \frac{B_{u}^{2}}{B^{2}}+\frac{\mathrm{I}}{3 A^{\prime \prime} B}\left[\frac{A_{v}^{\prime \prime}}{A^{\prime \prime}}+\frac{B_{v}}{B}\right], \\ A_{\mathrm{t}}^{\prime}=-\frac{A_{v}^{\prime \prime}}{3 A^{\prime \prime}}-\frac{B_{v}}{3 B}, \quad B_{\mathrm{t}}^{\prime}=\frac{B_{u}}{3 B}, \\ C_{1}^{\prime}=\frac{\mathrm{I}}{3}\left(A^{\prime \prime} B+\frac{\mathrm{I}}{A^{\prime \prime}}+\frac{\mathrm{I}}{B}\right)+\frac{B_{u}}{9 B}\left[\frac{A_{v}^{\prime \prime}}{A^{\prime \prime}}+\frac{B_{v}}{B}\right], \\ A_{1}^{\prime \prime}=B, \\ C_{\mathrm{t}}^{\prime \prime}=-\frac{\mathrm{I}}{3}\left[\frac{A_{v v}^{\prime \prime}}{A^{\prime \prime}}-\frac{A_{v}^{\prime \prime 2}}{A^{\prime \prime 2}}+\frac{B_{v v}}{B}-\frac{B_{v}^{2}}{B^{2}}\right]+\frac{2}{9}\left[\frac{A_{v}^{\prime \prime}}{A^{\prime \prime}}+\frac{B_{v}}{B}\right]^{2}-\frac{B_{u}}{3} .\end{array}\right.$

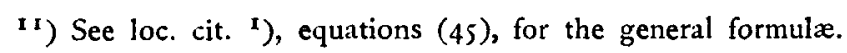


The invariants ${ }^{12}$ ) are found to be

(22)

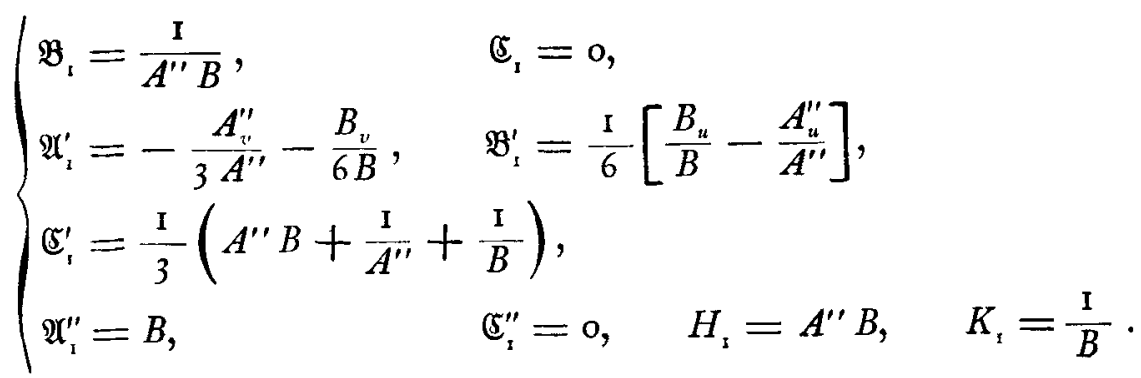

For the minus first LAPLACian transform we have ${ }^{\mathbf{1 3}}$ )

$$
\left\{\begin{array}{c}
A_{-1}=-B_{-1}^{\prime}, \quad B_{-1}=A^{\prime \prime} \\
C_{-\mathrm{I}}=-\frac{\mathrm{I}}{3}\left[\frac{A_{u u}^{\prime \prime}}{A^{\prime \prime}}-\frac{A_{u}^{\prime \prime 2}}{A^{\prime \prime 2}}+\frac{B_{u u}}{B}-\frac{B_{u}^{2}}{B^{2}}\right]+\frac{2}{9}\left[\frac{A_{u}^{\prime \prime}}{A^{\prime \prime}}+\frac{B_{u}}{B}\right]^{2}-\frac{A_{v}^{\prime \prime}}{3} \\
A_{-1}^{\prime}=\frac{A_{v \prime}^{\prime \prime}}{3 A^{\prime \prime}}, \cdot \quad B_{-1}^{\prime}=-\frac{\mathrm{I}}{3}\left[\frac{A_{u}^{\prime \prime}}{A^{\prime \prime}}+\frac{B_{u}}{B}\right] \\
C_{-\mathrm{I}}^{\prime}=\frac{\mathrm{I}}{3}\left(A^{\prime \prime} B+\frac{\mathrm{I}}{A^{\prime \prime}}+\frac{\mathrm{I}}{B}\right)+\frac{A_{v}^{\prime \prime}}{9 A^{\prime \prime}}\left[\frac{A_{u}^{\prime \prime}}{A^{\prime \prime}}+\frac{B_{u}}{B}\right] \\
A_{-1}^{\prime \prime}=\frac{\mathrm{I}}{A^{\prime \prime} B}, \quad B_{-1}^{\prime \prime}=-A_{-1}^{\prime} \\
C_{-1}^{\prime \prime}=\frac{A_{v v}^{\prime \prime}}{3 A^{\prime \prime}}-\frac{A_{v}^{\prime \prime 2}}{9 A^{\prime \prime 2}}+\frac{\mathrm{I}}{3 A^{\prime \prime} B}\left[\frac{A_{u}^{\prime \prime}}{A^{\prime \prime}}+\frac{B_{u}}{B}\right]
\end{array}\right.
$$

and

24) $\left\{\begin{array}{l}\mathfrak{B}_{-1}=A^{\prime \prime}, \quad \mathfrak{E}_{-1}=0, \\ \mathfrak{A}_{-1}^{\prime}=\frac{\mathrm{I}}{6}\left[\frac{A_{v}^{\prime \prime}}{A^{\prime \prime}}-\frac{B_{v}}{B}\right], \quad \mathfrak{B}_{-1}^{\prime}=-\frac{B_{u}}{3 B}-\frac{A_{u}^{\prime \prime}}{6 A^{\prime \prime}}, \quad \mathbb{E}_{-1}^{\prime}=\frac{\mathrm{I}}{3}\left(A^{\prime \prime} B+\frac{\mathrm{I}}{A^{\prime \prime}}+\frac{\mathrm{I}}{B}\right), \\ \mathfrak{U}_{-1}^{\prime \prime}=\frac{\mathrm{I}}{A^{\prime \prime} B}, \quad \mathbb{C}_{-\mathrm{r}}^{\prime \prime}=0, \quad H_{-1}=\frac{\mathrm{I}}{A^{\prime \prime}}, \quad K_{-\mathrm{s}}=A^{\prime \prime} B .\end{array}\right.$

The invariants and seminvariants of the first LAPLACian transform may be expressed interms of $A_{1}^{\prime \prime}$ and $B_{1}$ by equations exactly analogous to (17) and (20). Moreover, there exist conditions between $A_{1}^{\prime \prime}$ and $B_{\mathrm{r}}$ analogous to (r 8 ). The same thing is true for the minus first LapLAcian transform. Thus it may be verified analytically that any one of the three nets may be considered as the original net and that the other two would then be its Laplactan transforms. 


\section{\3. \\ The osculating conics of the three nets.}

Substituting the values of the invariants as given by (20) in equations (10) and (II), we find the equations of the conics which osculate the curves $v=$ const. and $u=$ const. respectively at $P_{y}$, to be

where

$$
\left\{\begin{array}{l}
M \equiv B^{2} x_{2}^{2}+\frac{4 B}{3}\left[\frac{A_{u}^{\prime \prime}}{A^{\prime \prime}}+\frac{B_{u}}{2 B}\right] x_{2} x_{3}-2 B x_{1} x_{3}+\varphi x_{3}^{2}=0, \\
N \equiv \psi x_{2}^{2}+\frac{4 A^{\prime \prime}}{3}\left[\frac{B_{v}}{B}+\frac{A_{v}^{\prime \prime}}{2 A^{\prime \prime}}\right] x_{2} x_{3}-2 A^{\prime \prime} x_{1} x_{2}+A^{\prime \prime 2} x_{3}^{2}=0,
\end{array}\right.
$$

$$
\left\{\begin{array}{l}
\varphi=\frac{2 A_{u u}^{\prime \prime}}{3 A^{\prime \prime}}-\frac{4 A_{u}^{\prime \prime 2}}{9 A^{\prime \prime 2}}+\frac{B_{u u}}{3 B}-\frac{4 B_{u}^{2}}{9 B^{2}}-\frac{A_{u}^{\prime \prime} B_{u}}{9 A^{\prime \prime} B}, \\
\psi=\frac{2 B_{v v}}{3 B}-\frac{4 B_{v}^{2}}{9 B^{2}}+\frac{A_{v v \prime}^{\prime \prime}}{3 A^{\prime \prime}}-\frac{4 A_{v}^{\prime \prime 2}}{9 A^{\prime \prime 2}}-\frac{A_{v}^{\prime \prime} B_{v}}{9 A^{\prime \prime} B} .
\end{array}\right.
$$

We can write down immediately the equations of the conics osculating the two curves of the $\sigma$-net which pass through $P_{\sigma}$, referred to a local coordinate system determined by the quantities $\sigma$ and the covariants of the $\sigma$-net which correspond to the covariants $p$ and $\sigma$ of the $y$-net. This coordinate system has the same triangle of reference as the local coordinate system of the $y$-net, but its sides are taken in different order and it has a different unit-point. We shall therefore have to transform the equations of the conics to the local coordinate system of the $y$-net before we shall be in a position to compare them with the equations (25).

Let $x_{1}^{(1)}, x_{2}^{(1)}, x_{3}^{(1)}$ be the coordinates of any point referred to the local coordinate system of the point $P_{\sigma}$ of the first Laplacian transformed net. Then the equations of the osculating conic of the curves $v=$ const. and $u=$ const. of the $\sigma$-net which meet at $P_{\sigma}$ will be

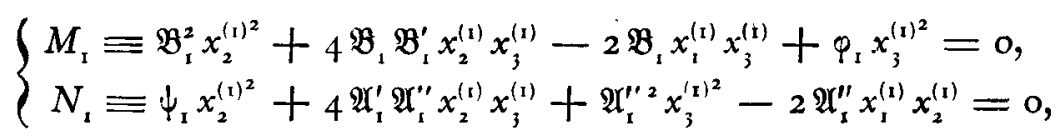

where $p_{1}$ and $\psi_{I}$ are defined by formula analogous to (II). We wish to find the equations of these conics referred to the local coordinate system of the $y$-net.

Let $y_{1}, p_{1}, \sigma_{1}$ represent the three covariants of the first Laplacian transformed net in its canonical form, corresponding respectively to $y, \rho, \sigma$ for the original net, as defined by $(20 a)$. We have the relation ${ }^{14}$ )

(28)

where

$$
\sigma=\lambda y_{1},
$$

$$
\lambda=\text { const. } \sqrt[3]{A^{\prime \prime} H} \text {. }
$$

14) loc. cit. " ), p. 488 . 
Making use of the equation (28) and (20 a) and the corresponding relations

$$
\rho_{1}=\frac{\partial y_{1}}{\partial u}-B_{1}^{\prime} y_{1}, \quad \sigma_{1}=\frac{\partial y_{1}}{\partial v}-A_{1}^{\prime} y_{1}
$$

for the first LAPLACIan transformed net we obtain the following equations:

$$
\lambda y_{\mathrm{I}}=\sigma, \quad \lambda \rho_{\mathrm{I}}=\frac{\mathrm{I}}{B} y, \quad \lambda \sigma_{\mathrm{I}}=A^{\prime \prime} \mathrm{p} .
$$

We are now ready to find the equations of transformation between the two systems of coordinates.

We have considered $x_{1}, x_{2}, x_{3}$ as the coordinates of a point referred to the local coordinate system of the $y$-net and $x_{1}^{(\mathrm{r})}, x_{2}^{(\mathrm{I})}, x_{3}^{(\mathrm{r})}$ as the coordinates of the same point with respect to the local coordinate system of the $\sigma$-net. Therefore we must have

$$
x_{1} y+x_{2} p+x_{3} \sigma=\omega\left(x_{1}^{(1)} y_{1}+x_{2}^{(1)} p_{1}+x_{3}^{(1)} \sigma_{1}\right)
$$

Making use of equations (29) this reduces to

$$
x_{1} y+x_{2} p+x_{3} \sigma=\omega\left(x_{1}^{(1)} \sigma+\frac{1}{B} x_{2}^{(1)} y+A^{\prime \prime} x_{3}^{(\mathrm{r})} \rho\right) \text {, }
$$

whence

$$
\omega x_{1}^{(1)}=x_{3}, \quad \omega x_{2}^{(1)}=B x_{1}, \quad \omega x_{3}^{(1)}=\frac{\mathrm{I}}{A^{\prime \prime}} x_{2} .
$$

From equations (21), (22), (27), (30) we obtain

where

$$
\left\{\begin{array}{l}
M_{1} \equiv x_{1}^{2}+\frac{2}{3}\left[\frac{B_{u}}{B}-\frac{A_{u}^{\prime \prime}}{A^{\prime \prime}}\right] x_{1} x_{2}-\frac{2}{B} x_{2} x_{3}+\varphi_{1} x_{2}^{2}=0 \\
N_{\mathrm{r}} \equiv \bigcup_{1} x_{1}^{2}-\frac{4}{3 A^{\prime \prime}}\left[\frac{A_{v}^{\prime \prime}}{A^{\prime \prime}}+\frac{B_{v}}{2 B}\right] x_{1} x_{2}-2 x_{1} x_{3}+\frac{\mathrm{I}}{A^{\prime \prime}-} x_{2}^{2}=0
\end{array}\right.
$$

$$
\left\{\begin{array}{l}
\varphi_{1}=\frac{B_{u u}}{3 B}-\frac{B_{u}^{2}}{9 B^{2}}-\frac{A_{u u}^{\prime \prime}}{3 A^{\prime \prime}}+\frac{2 A_{u}^{\prime \prime 2}}{9 A^{\prime \prime 2}}-\frac{A_{u}^{\prime \prime} B_{u}}{9 A^{\prime \prime} B}, \\
\psi_{1}=-\frac{2 A_{v v}^{\prime \prime}}{3 A^{\prime \prime}}+\frac{8 A_{v}^{\prime \prime 2}}{9 A^{\prime \prime 2}}-\frac{B_{v v}}{3}+\frac{5 B_{v}^{2}}{9 B^{2}}+\frac{5 A_{v}^{\prime \prime} B_{v}}{9 A^{\prime \prime} B} .
\end{array}\right.
$$

In a similar manner we obtain the equations of the osculating conics of the curves of the minus first Laplacian transform at $P_{g}$. They have the form

where

$$
\left\{\begin{array}{l}
M_{-1} \equiv \frac{\mathrm{I}}{B^{2}} x_{3}^{2}-\frac{4}{3 B}\left[\frac{B_{u}}{B}+\frac{A_{u}^{\prime \prime}}{2 A^{\prime \prime}}\right] x_{1} x_{3}-2 x_{1} x_{2}+\varphi_{-1} x_{\mathrm{r}}^{2}=0, \\
N_{-1} \equiv \psi_{-1} x_{3}^{2}+\frac{2}{3}\left[\frac{A_{v}^{\prime \prime}}{A^{\prime \prime}}-\frac{B_{v}}{B}\right] x_{1} x_{3}-\frac{2}{A^{\prime \prime}} x_{2} x_{3}+x_{1}^{2}=0,
\end{array}\right.
$$

$$
\left\{\begin{array}{l}
\varphi_{-1}=-\frac{2 B_{u t}}{3 B}+\frac{8 B_{u}^{2}}{9 B^{2}}-\frac{A_{u u}^{\prime \prime}}{3 A^{\prime \prime}}+\frac{5 A_{u}^{\prime \prime 2}}{9 A^{\prime \prime 2}}+\frac{5 A_{u}^{\prime \prime} B_{u}}{9 A^{\prime \prime} B} \\
\psi_{-1}=\frac{A_{v v}^{\prime \prime}}{3 A^{\prime \prime}}-\frac{A_{v}^{\prime \prime 2}}{9 A^{\prime \prime 2}}-\frac{B_{v^{v}}}{3 B}+\frac{2 B_{v}^{2}}{9 B^{2}}-\frac{A_{v}^{\prime \prime} B_{v}}{9 A^{\prime \prime} B} .
\end{array}\right.
$$

The developments of the next section show that in view of our choice of arbitrary functions and constants this may be considered as the most general set of six 
conics where one is tangent at each vertex of the triangle of reference to each of the two sides through that vertex. Six conics so related are not in general linearly dependent.

Let $\Delta$ represent the determinant of the sixth order formed by the coefficients of the conics $M, N, M_{1}, N_{\mathrm{I}}, M_{-\mathrm{r}}, N_{-\mathrm{r}}$ respectively. It may be reduced to the form

where

$$
\Delta=-A^{\prime \prime} B\left|a_{i j}\right|, \quad(i, j=1,2,3),
$$

$$
\begin{aligned}
& a_{11}=A^{\prime \prime}\left(\mathrm{I}-B^{2} \varphi_{-1} \psi_{-1}\right)\left[2 \frac{A_{u}^{\prime \prime}}{A^{\prime \prime}}+\frac{B_{u}}{B}\right]-B\left(\varphi \varphi_{-1}+A^{\prime \prime 2} \psi_{-1}\right), \\
& a_{12}=-B \varphi-A^{\prime \prime} B\left[2 \frac{A_{v}^{\prime \prime}}{A^{\prime \prime}}+\frac{B_{v}}{B}\right]-A^{\prime \prime} B^{2} \psi_{-1}\left[2 \frac{A_{u}^{\prime \prime}}{A^{\prime \prime}}+\frac{B_{u}}{B}\right], \\
& a_{13}=\mathrm{I}-A^{\prime \prime 2} B-A^{\prime \prime}\left[2 \frac{A_{u}^{\prime \prime}}{A^{\prime \prime}}+\frac{B_{u}}{B}\right]\left[\frac{A_{v}^{\prime \prime}}{A^{\prime \prime}}-\frac{B_{v}}{B}\right]-\varphi\left[\frac{A_{u}^{\prime \prime}}{A^{\prime \prime}}+2 \frac{B_{u}}{B}\right] \\
& \quad-A^{\prime \prime} B \psi_{-1}\left[2 \frac{A_{u}^{\prime \prime}}{A^{\prime \prime}}+\frac{B_{u}}{B}\right]\left[\frac{A_{u}^{\prime \prime}}{A^{\prime \prime}}+2 \frac{B_{u}}{B}\right], \\
& \quad-A^{\prime \prime} B \varphi_{1}\left[2 \frac{A_{v}^{\prime \prime}}{A^{\prime \prime}}+\frac{B_{v}}{B}\right]\left[\frac{A_{v}^{\prime \prime}}{A^{\prime \prime}}+2 \frac{B_{v}}{B}\right], \\
& a_{22}=\mathrm{I}-A^{\prime \prime} B^{2}+B\left[\frac{A_{v}^{\prime \prime}}{A^{\prime \prime}}+2 \frac{B_{v}}{B}\right]\left[\frac{A_{u}^{\prime \prime}}{A^{\prime \prime}}-\frac{B_{u}}{B}\right]-\psi\left[2 \frac{A_{v}^{\prime \prime}}{A^{\prime \prime}}+\frac{B_{v}}{B}\right] \\
& a_{23}=-A^{\prime \prime} \psi-A^{\prime \prime} B\left[\frac{A_{u}^{\prime \prime}}{A^{\prime \prime}}+2 \frac{B_{u}}{B}\right]-A^{\prime \prime 2} \psi_{\varphi_{1}}\left[\frac{A_{v}^{\prime \prime}}{A^{\prime \prime}}+2 \frac{B_{v}}{B}\right], \\
& a_{31}=B-A^{\prime \prime}+A^{\prime \prime} B^{2} \varphi_{-1} \psi_{-1}-A^{\prime \prime 2} B \varphi_{1} \psi_{1}, \\
& a_{32}=B\left[\frac{A_{u}^{\prime \prime}}{A^{\prime \prime}}-\frac{B_{u}}{B}\right]+A^{\prime \prime} B^{2} \psi_{-1}-B A^{\prime \prime} \varphi_{1}\left[2 \frac{A_{v}^{\prime \prime}}{A^{\prime \prime}}+\frac{B_{v}}{B}\right], \\
& a_{33}=A^{\prime \prime}\left[\frac{A_{v}^{\prime \prime}}{A^{\prime \prime}}-\frac{B_{v}}{B}\right]+A^{\prime \prime} B \psi_{-1}\left[\frac{A_{u}^{\prime \prime}}{A^{\prime \prime}}+2 \frac{B_{u}}{B}\right]-A^{\prime \prime 2} B \varphi_{1} \cdot
\end{aligned}
$$

Since neither family of our net may be straight lines, $A^{\prime \prime} \neq 0$ and $B \neq 0$. Hence the condition that the curves be linearly dependent is $\left|a_{i j}\right|=0$. Three special cases in which this condition is satisfied are $A^{\prime \prime}=B, A^{\prime \prime}=\mathrm{I} / B^{2}, B=\mathrm{I} / A^{\prime \prime 2}$, from which follows respectively, using (16), (22), and (24), $H=K, H_{1}=K_{1}, H_{-1}=K_{-1}$.

Since we may choose either of the three nets as the original net, each of these three cases mean the same thing, viz., equal Laplace-Darboux invariants for the original net. Professor WILCZYNSKI ${ }^{15}$ ) has shown that in this case the conics $M_{1}$ and

15) E. J. WiLczYNsk1, Über Flächen mit unbestimmten Direktrixkurven [Mathematische Annalen, Bd. LXXVI (1914), pp. I29-16o]. 
$N_{-1}$ coincide and the net may be considered as the stereographic projection on the directrix plane of the asymptotic curves of a surface with indeterminate directrix curves.

\section{$\$ 4$.}

\section{Determination of these nets by boundary conditions.}

The two invariants $A^{\prime \prime}$ and $B$, in terms of which all of the coefficients of the defining system of partial differential equations are expressed, satisfy the two partial differential equations of the second order

$$
\left\{\begin{array}{l}
\frac{\partial^{2} \log A^{\prime \prime}}{\partial u \partial v}=A^{\prime \prime} B-\frac{2}{A^{\prime \prime}}+\frac{\mathrm{I}}{B}, \\
\frac{\partial^{2} \log B}{\partial u \partial v}=A^{\prime \prime} B+\frac{\mathrm{I}}{A^{\prime \prime}}-\frac{2}{B} .
\end{array}\right.
$$

If we make the substitutions

$$
y_{1}=\log A^{\prime \prime}, \quad y_{2}=\log B
$$

we transform (r8) into

$$
\left\{\begin{array}{l}
\frac{\partial^{2} y_{1}}{\partial u \partial v}=e^{y_{1}+y_{2}}-2 e^{-y_{1}}+e^{-y_{2}}, \\
\frac{\partial^{2} y_{2}}{\partial u \partial v}=e^{y_{1}+y_{2}}+e^{-y_{1}}-2 e^{-y_{2}} .
\end{array}\right.
$$

It is easy to see that all of the derivatives of the form

$$
\frac{\partial^{\lambda+\mu} y_{i}}{\partial u^{\lambda} \partial v^{\mu}}, \quad(\lambda \neq 0, \mu \neq 0)
$$

may be obtained from $(36)$ in terms of derivatives of lower order while the values of all the derivatives of the form

$$
\frac{\partial^{\lambda} y_{i}}{\partial u^{2}}, \quad \frac{\partial^{\mu} y_{i}}{\partial v^{\mu}}
$$

remain arbitrary. Consequently a simple application of familiar existence theorems teaches us that (36) has analytic solutions $y_{1}$ and $y_{2}$ which, for $v=v_{0}$, reduce to

$$
y_{1}=\log A^{\prime \prime}=U_{1}(u), \quad y_{2}=\log B=U_{2}(u),
$$

where $U_{1}$ and $U_{2}$ are arbitrary analytic functions of $u$ alone, while for $u=u_{0}, y_{\text {s }}$ and $y_{2}$ reduce to

$$
y_{1}=V_{1}(v), \quad y_{2}=V_{2}(v),
$$

where $V_{1}$ and $V_{2}$ are arbitrary analytic functions of $v$ alone, and where, of course, we must have

(40)

$$
U_{1}\left(u_{0}\right)=V_{1}\left(v_{0}\right), \quad U_{2}\left(u_{0}\right)=V_{2}\left(v_{0}\right)
$$

The four arbitrary functions, each of a single variable, which we have at our 
disposal may be chosen so as to make a curve $u=$ const. and a curve $v=$ const. of the original net, a curve $v=$ const. of the first Laplacian transformed net and a curve $u=$ const. of the minus first Laplacian transformed net coincide with four arbitrarily chosen curves. We shall now prove this statement and show further that the net will then be uniquely determined except for six arbitrary constants.

We first recall a fundamental theorem of the projective differential geometry ${ }^{16}$ ) of plane curves. Let $y_{1}(x), y_{2}(x), y_{3}(x)$ be homogeneous coordinates of a point $P$ which describes a non-rectilinear, analytic, plane curve as $x$ varies. Then $y_{1}, y_{2}, y_{3}$ are linearly independent solutions of a linear homogeneous differential equation of the third order, viz :

$$
\frac{d^{3} y}{d x^{3}}+3 p_{1} \frac{d^{2} y}{d x^{2}}+3 p_{2} \frac{d y}{d x}+p_{3} y=0,
$$

whose seminvariants

$$
P_{2}=p_{2}-p_{1}^{2}-p_{1}^{\prime}, \quad P_{3}=p_{3}-3 p_{1} p_{2}+2 p_{\mathrm{I}}^{3}-p_{\mathrm{I}}^{\prime \prime}
$$

and invariants

$$
\theta_{3}=P_{3}-\frac{3}{2} P_{2}^{\prime}, \quad \theta_{8}=6 \theta_{3} \theta_{3}^{\prime \prime}-7\left(\theta_{3}^{\prime}\right)^{2}-27 P_{2} \theta_{3}^{2}
$$

may be determined as functions of $x$ if $y_{1}, y_{2}, y_{3}$ are given.

On the other hand, if two arbitrary functions $\varphi_{3}(x)$ and $\phi_{8}(x)$ be given there exists a curve whose invariants $\theta_{3}$ and $\theta_{8}$ are respectively equal to these given functions and this curve will be uniquely determined except for projective transformations. In particular, if $\theta_{3}=0$ the corresponding curve is a conic.

If we differentiate both members of the first equation of (19) with respect to $u$ and then eliminate $y_{v}$ and $y_{u v}$, we obtain the following equation:

where

$$
y_{u u u}+3 q_{1} y_{u u}+3 q_{2} y_{u}+q_{3} y=0,
$$

$$
\begin{aligned}
& q_{\mathrm{s}}=-\frac{B_{u}}{3 B}, \quad q_{2}=-\frac{A_{u}^{\prime \prime}}{9 A^{\prime \prime}}\left[\frac{A_{u}^{\prime \prime}}{A^{\prime \prime}}+\frac{B_{u}}{B}\right], \\
& q_{3}=-\frac{A_{u u u}^{\prime \prime}}{3 A^{\prime \prime}}+\frac{2 A_{u}^{\prime \prime} A_{u u}^{\prime \prime}}{3 A^{\prime 22}}-\frac{7 A_{u}^{\prime \prime 3}}{27 A^{\prime 3}}+\frac{A_{u}^{\prime \prime} B_{u}}{3 A^{\prime \prime} B}-\frac{A_{u}^{\prime \prime 2} B_{u}}{9 A^{\prime 2} B}-\mathrm{r} .
\end{aligned}
$$

If we put $v=v_{0}$ in the coefficients, $(4 \mathrm{I} a)$ is the differential equation of the curve $v=v_{\mathrm{o}}$ of the original net. For $v=v_{\mathrm{o}}, y, A^{\prime \prime}$ and $B$ are functions of $u$ alone and this may be regarded as an ordinary differential equation. We compute by formulæ analogous to $(42)$ and $(43)$ the invariants $\theta_{3}(u)$ and $\theta_{8}(u)$. After making the convenient substitution

$$
U_{1}(u)=\log A^{\prime \prime}, \quad U_{2}(u)=\log B,
$$

16) E. J. Wilczynski, Projective Differential Geometry of Curves and Ruled Surfaces (Leipzig, B. G. Teubner, I906), pp. $58-6 \mathrm{I}$. 
they take the form

(44)

$$
\left\{\begin{array}{c}
\theta_{3}=\frac{1}{54}\left[-18 U_{1}^{\prime \prime \prime}-9 U_{2}^{\prime \prime \prime}+27 U_{1}^{\prime \prime} U_{2}^{\prime}+9 U_{1}^{\prime} U_{2}^{\prime \prime}+18 U_{2}^{\prime} U_{2}^{\prime \prime}\right. \\
\quad+4\left(U_{1}^{\prime 3}-U_{2}^{\prime 3}\right)+6 U_{1}^{\prime} U_{2}^{\prime}\left(U_{1}^{\prime}\right. \\
\theta_{8}=6 \theta_{3} \theta_{3}^{\prime \prime}-7 \theta_{3}^{\prime 2}-27 Q_{2} \theta_{3}^{2},
\end{array}\right.
$$

where

$$
Q_{2}=\frac{1}{9}\left(3 U_{2}^{\prime \prime}-U_{1}^{\prime 2}-U_{1}^{\prime} U_{2}^{\prime}-U_{2}^{\prime 2}\right) \text {. }
$$

In a similar manner we obtain the invariants for a curve $v=v_{0}$ of the minus first LAPLAClan transform in the form

$$
\left(\theta_{8}^{(-1)}=6 \theta_{3}^{(-1)} \theta_{3}^{(-1)^{\prime \prime}}-7\left(\theta_{3}^{(-1)^{\prime}}\right)^{2}-27 Q_{2}^{(-1)}\left(\theta_{3}^{(-1)}\right)^{2},\right.
$$

where

$$
Q_{2}^{(-1)}=\frac{1}{9}\left(3 U_{1}^{\prime \prime}-U_{1}^{\prime 2}-U_{1}^{\prime} U_{2}^{\prime}-U_{2}^{\prime 2}\right) \text {. }
$$

The theorem concerning the existence of solutions of (36) assures us that $U_{1}$ and $U_{2}$ may be chosen as arbitrary functions of $u$. We shall now prove that these arbitrary functions $U_{1}$ and $U_{2}$ can be chosen in such a way as to make the curve $v=v_{0}$ of the original net and the corresponding curve $v=v_{0}$ of the minus first LAPLACIan transform coincide with two arbitrarily chosen non-rectilinear analytic curves.

Let $C$ be any analytic curve, not a straight line, and let equation (4I) be its differential equation with respect to any independent variable $x$ to which it may be referred parametrically. Let $\theta_{3}=\varphi_{3}(x)$ and $\theta_{\mathrm{s}}=p_{8}(x)$ be the values of its invariants as functions of $x$. Further, let $C_{-1}$ be any other such curve having a differential equation analogous to $(4 \mathrm{I})$ referred to another variable $\xi$ as a parameter. Let $\theta_{3}^{(-1)}-\varphi_{3}^{(-1)}(\xi)$, $\theta_{8}^{(-1)}=\varphi_{8}^{(-1)}(\xi)$ be the values of its invariants as functions of $\xi$. If the curve $v=v_{\mathrm{o}}$ of the original net and the corresponding curve $v=v_{0}$ of the minus first Laplacian transform coincide with $C$ and $C_{-r}$ respectively, it must be possible to determine $u$ as a function of $x$ and $\xi$ as a function of $x$, and consequently $u$ as a function of $\xi$, so that the equations

$$
\left\{\begin{array}{c}
\theta_{3}(u)=\varphi_{3}(x), \\
\theta_{8}(u)=\varphi_{8}(x), \\
\theta_{3}^{(-1)}(u)=\varphi_{3}^{(-1)}(\xi), \\
\theta_{8}^{(-1)}(u)=\varphi_{8}^{(-1)}(\xi),
\end{array}\right.
$$

will be identically satisfied.

Conversely, if equations (46) are satisfied the curves $C$ and $C_{-1}$ will be projectively equivalent to the curves $v=v_{0}$ of the original net and of the minus first $\mathrm{LA}_{\mathrm{A}}$ PLAcian transform respectively. By choosing the fundamental system of solutions of the differential equation the curves may be made to coincide.

To reduce the order of the differential equations which will appear in this pro- 
blem we make the substitution

(47)

$$
w=U_{1}^{\prime}, \quad z=U_{2}^{\prime}
$$

in (44) and (45). Substituting then the resulting expressions for $\theta_{3}, \theta_{8}, \theta_{3}^{(-5)}, \theta_{8}^{(-1)}$ in (46) we find that the differential equations to be satisfied by $y, z, u$ and $\xi$ as functions of $x$ have the form

(48)

$$
\left\{\begin{array}{c}
-18 \frac{d^{2} w}{d u^{2}}-9 \frac{d^{2} z}{d u^{2}}+27 z \frac{d w}{d u}+9(w+2 z) \frac{d z}{d u} \\
+2(w-z)(2 w+z)(w+2 z)-54=54 \varphi_{3}(x), \\
6 \varphi_{3} \frac{d^{2} \varphi_{3}}{d u^{2}}-7\left(\frac{d \varphi_{3}}{d u}\right)^{2}-3\left(\frac{d z}{d u}-w^{2}-w z-z^{2}\right) \varphi_{3}^{2}=\varphi_{8}(x), \\
9 \frac{d^{2} w}{d u^{2}}+18 \frac{d^{2} z}{d u^{2}}-9(2 w+z) \frac{d w}{d u}-27 w \frac{d z}{d u} \\
+2(w-z)(2 w+z)(w+2 z)-54=54 \varphi_{3}^{(-1)}(\xi), \\
6 \varphi_{3}^{(-1)} \frac{d^{2} \varphi_{3}^{(-1)}}{d u^{2}}-7\left(\frac{d \varphi_{3}^{(-1)}}{d u}\right)^{2}-3\left(\frac{d w}{d u}-w^{2}-w z-z^{2}\right) \varphi_{3}^{(-1)^{2}}=\varphi_{8}^{(-1)}(\xi) .
\end{array}\right.
$$

If $C$ be not a conic, so that $\varphi_{3}(x) \neq 0$, we can, by a suitably chosen transformation of the independent variable $x$, make $\left.\varphi_{3}(x)=\mathrm{I}^{{ }^{17}}\right)$ and similarly for $\varphi_{3}^{(-1)}(\xi)$. Assuming these transformations made and using $x$ as the independent variable in all of the differentiations which occur in equations (48), we may write the equations in the form

$$
\begin{aligned}
& -\mathrm{I} 8 \frac{d}{d x}\left[\frac{\frac{d w}{d x}}{\frac{d u}{d x}}\right] \frac{1}{\frac{d u}{d x}}-9 \frac{d}{d x}\left[\frac{\frac{d z}{d x}}{\frac{d u}{d x}}\right] \frac{\mathrm{I}}{\frac{d u}{d x}}+27 z \frac{\frac{d w}{d x}}{\frac{d u}{d x}}+9(w+2 z) \frac{\frac{d z}{d x}}{\frac{d u}{d x}} \\
& +2(w-z)(w+2 z)(2 w+z)-108=0 \text {, } \\
& 3 \frac{d}{d x}\left[\frac{\frac{d z}{d x}}{\frac{d u}{d x}}\right]-\left(w^{2}+w z+z^{2}\right)=-\frac{1}{3} \varphi_{8}(x) \\
& 9 \frac{d}{d x}\left[\frac{\frac{d w}{d x}}{\frac{d u}{d x}}\right] \frac{\mathrm{I}}{\frac{d u}{d x}}+18 \frac{d}{d x}\left[\frac{\frac{d z}{d x}}{\frac{d u}{d x}}\right] \frac{\mathrm{I}}{\frac{d u}{d x}}-9(2 w+z) \frac{\frac{d w}{d x}}{\frac{d u}{d x}}-27 w \frac{\frac{d z}{d x}}{\frac{d u}{d x}} \\
& +2(w-z)(w+2 z)(2 w+z)-108=0 \text {, } \\
& 3 \frac{d}{d x}\left[\frac{\frac{d w}{d x}}{\frac{d u}{d x}}\right]-\left(w^{2}+w z+z^{2}\right)=-\frac{\mathrm{I}}{3} \varphi_{8}^{-1}(\xi) \text {. }
\end{aligned}
$$

17) loc. cit. $\left.{ }^{16}\right)$, p. 6 I. 
Using the second and fourth equation to reduce the first and third we obtain the final form

(50)

$$
\left\{\begin{array}{c}
{\left[7 w^{3}+24 w^{2} z+12 w z^{2}+11 z^{3}-108-3 z \varphi_{8}^{-1}-(w+2 z) \varphi_{8}\right] \frac{d u}{d x}} \\
=(w+z)\left(w^{2}+w z+z^{2}\right)-2 \frac{d \varphi_{8}^{-1}}{d \xi} \frac{d \xi}{d x}-\frac{d \varphi_{8}}{d x}-(2 w+z) \varphi_{8}^{-1}-(w+2 z) \varphi_{8} \\
9 \frac{d w}{d x}=\left[3\left(w^{2}+w z+z^{2}\right)-\varphi_{8}(x)\right] \frac{d u}{d x}, \\
{\left[\mathrm{II} w^{3}+12 w^{2} z+24 w z^{2}+7 z^{3}-108-(2 w+z) \varphi_{8}^{-1}-3 w \varphi_{8}\right] \frac{d u}{d x}} \\
=(w+z)\left(w^{2}+w z+z^{2}\right)-\frac{d \varphi_{8}^{-1}}{d \xi} \frac{d \xi}{d x}-2 \frac{d \varphi_{8}}{d x}-(z+2 w) \varphi_{8}^{-1}-(w+2 z) \varphi_{8} \\
9 \frac{d z}{d x}=\left[3\left(w^{2}+w z+z^{2}\right)-\varphi_{8}^{-1}(\xi)\right] \frac{d u}{d x} .
\end{array}\right.
$$

We have in (5o) four differential equations of the first order with four dependent variables $w, z, u$, and $\xi$ and the independent variable $x$. According to the well known existence theorems for ordinary differential equations there exist four integrals involving the variables and four independent arbitrary constants from which can be determined $u, z, u$ and $\xi$ as functions of $x$ and the four constants.

Inasmuch as the variable $u$ itself does not occur in system (50), but only its derivative $d u / d x, u+k$ will satisfy the same system as that satisfied by $u$ if $k$ is an arbitrary constant. This constant may be chosen equal to zero without loss of generality by fixing arbitrarily the origin of the $u$-scale.

Let us select a point $u=u_{\mathrm{o}}$ on the curve $C$. Since the point $P_{\mathrm{p}}$ must be on the line tangent to the curve $C$ at $P_{y}$, it can occupy only a single infinity of positions. If the curve $C_{-1}$ is prescribed, the position of the point $P_{p}$ on the tangent will thereby be determined thus giving to a single relation between the three remaining arbitrary constants arising from the integration of equations (50).

We have further

so that

$$
U_{1}^{\prime}=w, \quad U_{2}^{\prime}=z,
$$

$$
U_{\mathrm{r}}=\int w d u+\text { const., } \quad U_{2}=\int z d u+\text { const., }
$$

whence, in view of (47),

$$
A^{\prime \prime}=\text { const. } e^{\int_{w i t u}}, \quad B=\text { const. } e^{\int_{z d u}} .
$$

Hence the functions $U_{1}(u)$ and $U_{2}(u)$ are determined except for four arbitrary constants. In a similar manner we determine two functions

$$
V_{\mathrm{s}}(v)=\frac{A_{v}^{\prime \prime}}{A^{\prime \prime}}, \quad V_{2}(v)=\frac{B_{v}}{B},
$$

involving four arbitrary constants by means of the invariants of the arbitrarily chosen curves $u=u_{\mathrm{o}}$ of the original net and $u=u_{\mathrm{o}}$ of the first LApLAcian transform, and 
by choosing a definite point of the tangent as the position of the point $P_{\sigma}$ of the first Laplacian transformed net which shall correspond to $P_{y}$. By means of the conditions (40) the eight constants reduce to six.

Two of the arbitrary constants occur in $A_{u}^{\prime \prime} / A^{\prime \prime}$ and $B_{u} / B$, two in $A_{v}^{\prime \prime} / A^{\prime \prime}$ and $B_{v} / B$; the two remaining ate factors respectively of $A^{\prime \prime}$ and $B$.

We see by inspection of (3I) and (33) that the conic $M_{1}$ contains three of these arbitrary constants in its coefficients while the conic $N_{-1}$ contains the other three. Since the conic $N_{-1}$ must touch at $P_{\rho}$ the line tangent to the curve $C$ at $P_{y}$ and since all such conics form a 3-parameter family, we may think of the determination of the three constants as corresponding geometricall $l_{j}$ to the selection of a specific conic of this 3-parameter family as the conic $N_{-1}$. In a similar manner we determine a conic $M_{1}$ and the other three constarits.

We consider now the case when $\varphi_{3}(x)=\phi_{3}^{-1}(\xi)=o$, i. e., when the curves of the nets are conics. From (48) we find in this case

where

$$
\left\{\begin{array}{r}
-18 \frac{d^{2} w}{d u^{2}}-9 \frac{d^{2} z}{d u^{2}}+27 z \frac{d w}{d u}+9(w+2 z) \frac{d z}{d u}+f(w, z)=0 \\
9 \frac{d^{2} w}{d u^{2}}+18 \frac{d^{2} z}{d u^{2}}-9(2 w+z) \frac{d w}{d u}-18 w \frac{d z}{d u}+f(w, z)=0
\end{array}\right.
$$

$$
f(w, z)=4 w^{3}+6 w^{2} z+6 w z^{2}-4 z^{3}-54 .
$$

Subtracting the corresponding members of the second equation from those of the first and integrating the exact differential thus formed we obtain

$$
-3 \frac{d w}{d u}-3 \frac{d z}{d u}+w^{2}+4 w z+z^{2}+c_{1}=0,
$$

where $c_{\mathrm{r}}$ is the constant of integration. From equations $(48 a)$ and $(5 \mathrm{I})$ we obtain, after performing a quadrature,

$$
9\left[\frac{d(w-z)}{d u}\right]^{2}=(w-z)^{4}+6 c_{1}(w-z)^{2}+216(w-z)+c_{2}
$$

showing that in this case $w-z$ may be expressed as an elliptic function of $u$. The additive constant of integration which occurs may be chosen equal to zero without loss of generality by properly selecting the origin of the $u$-scale. We have then as a solution of (5I) and (52) $w$ and $z$ as functions of $u$ and three arbitrary constants. The determination of these and of the remaining constants of integration may be made as in the more general case discussed before.

We have obtained, therefore, the following theorem:

Choose an arbitrary triangle $L M N$. Through the point $L$ pass two non rectilinear but otherwise arbitrary analytic curves $C$ and $C^{\prime}$ tangent to $L N$ and $L M$ respectively at $L$. Through each of the points $M$ and $N$ pass another such arbitrary curve, $C_{1}^{\prime}$ through $M$ and $C_{-1}$ through $N$, tangent to $M N$ at $M$ and $N$ respectively. Select any conic $M_{\text {, }}$ tangent to $L M$ at $M$ and a second conic $N_{-1}$ tangent to $L N$ at $N$. There exists one 
and only one net periodic of period 3 under the LAPLACIan transformation which contains $C$ and $C^{\prime}$, which has $C_{-1}$ corresponding to $C$ and $C_{1}^{\prime}$ corresponding to $C^{\prime}$ as curves of the minus first and first LAPLACian transforms respectively, and which moreover bas $M_{\mathrm{I}}$ as the osculating conic of the curve of the first LAPLACIan transform corresponding to $C$ and $N_{-1}$ as the osculating conic of the curve of the minus first LAPLACian transform corresponding to $C^{\prime}$.

\section{\$5.}

\section{Nets of conics whose Laplacian transforms are coincident straight lines.}

The special case which arises when the conditions

$$
H=K=\mathrm{o}
$$

are added to $\mathfrak{C}=\mathfrak{C}^{\prime \prime}=\mathrm{o}$ is of great interest in connection with the theory of surfaces and has been studied in its general aspects by Professor WilczYnski. We shall discuss in considerable detail the still more special case, when all of these conditions are satisfied and when besides all of the curves of the net are conics.

Since a net for which $H=K=0$ has degenerate Laplacian transforms ${ }^{18}$ ), it is not justifiable in this case to speak of the net as being periodic. We interpret geometrically the conditions $\mathfrak{E}=\mathfrak{S}^{\prime \prime}=0$ as follows.

If $\mathbb{E}=\mathfrak{E}^{\prime \prime}=\mathrm{o}$ simultaneously with the conditions (53) the two curves into which the transformed nets degenerate become straight lines and are coincident. In the first place the conditions ${ }^{19}$ ).

$$
\mathfrak{A}^{\prime \prime 2} K+\mathfrak{A}^{\prime \prime} \mathfrak{C}_{v}^{\prime \prime}-\mathfrak{S}^{\prime \prime} \mathfrak{A}_{v}^{\prime \prime}=\mathrm{o}, \quad \mathfrak{B}^{2} H+\mathfrak{B} \mathfrak{C}_{u}-\mathfrak{C} \mathfrak{B}_{u}=\mathrm{o}
$$

that the degenerate Laplacian transforms be straight lines are identically satisfied on account of (53) and (12). To show that these two lines coincide we return to the fundamental geometrical interpretation of the conditions $\left.\mathfrak{E}=\mathfrak{E}^{\prime \prime}=0^{20}\right)$. If $\mathfrak{E}=0$, the curve described by $P_{p}$ as $P_{y}$ moves along a curve $v=$ const. is such that its tangent at $P_{\rho}$ passes through $P_{\sigma}$. If $\mathbb{C}^{\prime \prime}=0$, the curve described by $P_{\sigma}$ as $P_{y}$ moves along a curve $u=$ const. is such that its tangent at $P_{\sigma}$ passes through $P_{\rho}$. The tangents to the curves of the LAPLACran transforms in our case are the two straight lines themselves and they have the points $P_{\rho}$ and $P_{\sigma}$ in common.

The form of the coefficients of the system of partial differential equations which satisfy the conditions

$$
\mathfrak{c}=\mathfrak{c}^{\prime \prime}=\mathrm{o}, \quad H=K
$$

18) The p-net and o-net each degenerate into a single curve and this curve represents the family of direct transforms. See loc. cit. ${ }^{\mathrm{I}}$ ), pp. $490,493$.

19) loc. cit. ${ }^{\mathrm{I}}$ ), equations ( $\left.54 a\right),(54 b)$.

20) loc. cit. ${ }^{\mathbf{I}}$ ), p. 506 . 
has been determined by Professor WILCZYNSKI ${ }^{21}$ ). If in particular we assume $H=K=0$ we must equate to zero the quantity $k$ which occurs in his formula and we obtain the equations

where

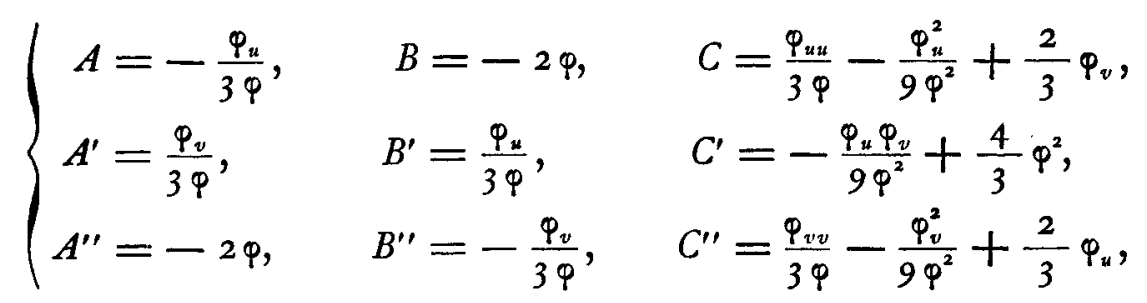

$$
\left.\varphi={\frac{\sqrt{U^{\prime} V^{\prime}}}{2(U+V)}}^{22}\right)
$$

$U$ and $V$ being arbitrary functions of $u$ alone and $v$ alone respectively. The invariants have the form

$$
\left\{\begin{aligned}
\mathfrak{B} & =-2 \varphi, & \mathfrak{S} & =0, \\
\mathfrak{A}^{\prime} & =\frac{\varphi_{v}}{2 \varphi}, & \mathfrak{B}^{\prime} & =\frac{\varphi_{u}}{2 \varphi}, \\
\mathfrak{U}^{\prime \prime} & =-2 \varphi, & \mathfrak{S}^{\prime \prime} & =0 .
\end{aligned}\right.
$$

The condition that the curves $v=$ const. and the curves $u=$ const., respectively, be conics ${ }^{23}$ ) may be written

$$
\left\{\begin{aligned}
3 B \bar{\varphi}_{u}-4 B_{u} \bar{\varphi}-6 B B^{\prime} \bar{\varphi}+6 B^{2} H & =0 \\
3 A^{\prime \prime} \psi_{v}-4 A_{v}^{\prime \prime} \psi-6 A^{\prime} A^{\prime \prime} \psi+6 A^{\prime \prime} & =0
\end{aligned}\right.
$$

where $\bar{\varphi}$ and $\psi$ are defined by (II). Making use of equations (53), (54), (56) the first equation of $(57)$ reduces to

$$
\frac{\partial^{3} \log \varphi}{\partial u^{3}}-2 \frac{\partial \log \varphi}{\partial u} \cdot \frac{\partial^{2} \log \varphi}{\partial u^{2}}=0 .
$$

From (55) we obtain by differentiation

$$
\left\{\begin{array}{l}
\frac{\partial \log \varphi}{\partial u}=\frac{1}{2}\left[\frac{U^{\prime \prime}}{U^{\prime}}-2 \frac{U^{\prime}}{U+V}\right], \\
\frac{\partial^{2} \log \varphi}{\partial u^{2}}=\frac{1}{2}\left[\frac{U^{\prime \prime \prime}}{U^{\prime}}-\frac{U^{\prime \prime}}{U^{\prime 2}}-\frac{2 U^{\prime \prime}}{U+V}+\frac{2 U^{\prime 2}}{(U+V)^{2}}\right] .
\end{array}\right.
$$

Substituting the second members of $(59)$ in $(58)$ we obtain the equation

where

$$
\frac{d}{d u}\{U, u\}=0 \text {, }
$$

$$
\{U, u\}=\frac{U^{\prime \prime \prime}}{U^{\prime}}-\frac{3 U^{\prime \prime 2}}{2 U^{\prime 2}}
$$

21) loc. cit. 15), p. 139, equations (35).

22) loc. cit. ${ }^{15}$ ), p. I35.

23) loc. cit. '), p. 507. 
denotes the Schwarzian derivative of $U$ with respect to $u$. This gives on integration

$$
U=c_{3} \tan \left(c_{1} u+c_{2}\right)+c_{4},
$$

where $c_{1}, c_{2}, c_{3}, c_{4}$ are the constants of integration. In a similar manner we find

$$
V=d_{3} \tan \left(d_{\mathrm{r}} v+d_{2}\right)+d_{4} \text {. }
$$

We may make the further transformation of the independent parameters

$$
\bar{u}=c_{1} u+c_{2}, \quad \bar{v}=c_{1} v+\frac{c_{1} d_{2}}{d_{1}}
$$

without disturbing the canonical form indicated by the form (54) of the coefficients ${ }^{24}$ ). Thus we may write

$$
\left\{\begin{array}{l}
U=c_{3} \tan u+c_{4}, \\
V=d_{3} \tan \frac{d_{1}}{c_{1}} v+d_{4} .
\end{array}\right.
$$

Substituting (60) in (55) we find

$$
\varphi=\frac{1}{2} \frac{\sqrt{c_{3} d_{3} c_{1} / d_{1}} \sec u \sec v}{c_{3} \tan u+d_{3} \tan \frac{d_{1}}{c_{1}} v+c_{4}+d_{4}},
$$

which reduces finally to the form

$$
\varphi=\frac{1}{2} \frac{\sqrt{p q}}{p \sin u \cos q v+\cos u \sin q v+r \cos u \cos q v},
$$

where $p=c_{3} / d_{3}, q=\frac{d_{1}}{c_{1}}, r=\left(c_{4}+d_{4}\right) / d_{3}$ are the three independent arbitrary constants remaining.

Thus we see that there exist precisely $\infty^{3}$ projectively distinct classes of nets which are composed of conics and which satisfy besides the four conditions $H=K=\mathfrak{C}=\mathfrak{C}^{\prime \prime}=0$.

Let

$$
\Delta(u, v)=p \sin u \cos q v+\cos u \sin q v+r \cos u \cos q v .
$$

We may write our seminvariants $(54)$ in the form
(63) $\left\{\begin{array}{c}A=\frac{\Delta_{u}}{3 \Delta}, \\ A^{\prime}=-\frac{\Delta_{v}}{3 \Delta}, \\ A^{\prime \prime}=-\frac{\sqrt{p q}}{\Delta},\end{array}\right.$

$$
\begin{aligned}
& B=-\frac{\sqrt{p q}}{\Delta}, \\
& B^{\prime}=-\frac{\Delta_{u}}{3 \Delta},
\end{aligned}
$$$$
C=-\frac{\Delta_{u u}}{3 \Delta}+\frac{5 \Delta_{u}^{2}}{9 \Delta^{2}}-\frac{\sqrt{p q} \Delta_{v}}{3 \Delta^{2}}
$$$$
C^{\prime}=-\frac{\Delta_{u v}}{3 \Delta}+\frac{2 \Delta_{u} \Delta_{v}}{9 \Delta^{2}}
$$$$
B^{\prime \prime}=\frac{\Delta_{v}}{3 \Delta},
$$$$
C^{\prime \prime}=-\frac{\Delta_{v v}}{3 \Delta}+\frac{5 \Delta_{v}^{2}}{9 \Delta^{2}}-\frac{\sqrt{p q_{u}} \Delta_{u}}{3 \Delta^{2}} \text {. }
$$

On the other hand, if we consider a system of partial differential equations whose coefficients are defined by $\left(6_{2}\right)$ and $\left(6_{3}\right)$ we find the conditions $H=K=\mathfrak{C}=\mathfrak{C}^{\prime \prime}=\mathrm{o}$

24) loc. cit. ${ }^{15}$ ), p. I33, equations (14). 
and the integrability conditions identically satisfied and besides the net defined by three independent solutions of such a system is a net of conics.

We proceed to integrate the system of partial differential equations of the net. First we make the transformation

$$
y=-\left(\frac{\sqrt{p q}}{\Delta}\right)^{\frac{1}{3}} \bar{y}
$$

on the system with coefficients defined by (63). Indicating by $\bar{a}, \bar{b}, \bar{c}$, etc., the coefficients of the transformed system, we have

$$
\bar{a}=\frac{\Delta_{u}}{\Delta}, \quad \bar{b}=\bar{a}^{\prime \prime}=-\frac{\sqrt{p q}}{\Delta}, \quad \overline{b^{\prime \prime}}=\frac{\Delta_{v}}{\Delta}, \quad \overline{a^{\prime}}=\overline{b^{\prime}}=\overline{c^{\prime}}=\bar{c}=\overline{c^{\prime \prime}}=0 .
$$

We have then to integrate a system of partial differential equations of the form

$$
\left\{\begin{array}{l}
y_{u u}=\frac{\Delta_{u}}{\Delta} y_{u}-\frac{\sqrt{p q}}{\Delta} y_{v} \\
y_{u v}=0 \\
y_{v v}=-\frac{\sqrt{p q}}{\Delta} y_{u}+\frac{\Delta_{v}}{\Delta} y_{v}
\end{array}\right.
$$

where $\Delta$ is defined by (62). We find the following three linearly independent solutions of this system

$$
\left\{\begin{array}{l}
y_{1}=\sin u-\frac{P}{r} \cos u+\frac{\sqrt{p}}{q r \sqrt{q}} \cos q v, \\
y_{2}=\frac{q \sqrt{p q}}{r} \cos u+\sin q v-\frac{\mathrm{I}}{r} \cos q v, \\
y_{3}=\mathrm{I} .
\end{array}\right.
$$

The equation of the family of curves $v=$ const. has the form

$$
\left\{\begin{array}{l}
p q^{3} x_{\mathrm{x}}^{2}+\left(p^{2}+r^{2}\right) x_{2}^{2}+\left[\left(p^{2}+r^{2}\right) \sin ^{2} q v-2 r \sin q v \cos q v+\cos ^{2} q v-p q^{3}\right] x_{3}^{2} \\
+2 \sqrt{p^{3} q^{3}} x_{1} x_{2}-2 \sqrt{p^{3} q^{3}} \sin q v x_{1} x_{3}-2\left[\left(q^{2}+r^{2}\right) \sin q v-r \cos q v\right] x_{2} x_{3}=0,
\end{array}\right.
$$

and for the family of curves $u=$ const. we find the form

$$
\text { (67) }\left\{\begin{array}{l}
q^{3}\left(r^{2}+1\right) x_{1}^{2}+p x_{2}^{2}+q^{3}\left[\left(r^{2}+1\right) \sin ^{2} u-2 p r \sin u \cos u+p^{2} \cos ^{2} u-\frac{p}{q_{3}^{3}}\right] x_{3}^{2} \\
+2 q \sqrt{p q} x_{1} x_{2}-2 \sqrt{p q^{3}} \sin u x_{2} x_{3}-2 q^{3}\left[\left(r^{2}+1\right) \sin u-p r \cos u\right] x_{1} x_{3}=0 .
\end{array}\right.
$$
forms.

The line $x_{3}=0$ is at the same time the first and minus first Laplacian trans-

It is possible to determine (except for projective transformations) a net of the kind under discussion by choosing an arbitrary line as the LApLAcian transform and two arbitrary conics as curves of the net. We shall need the equations of the curves of the net referred to the local triangle of reference $P_{y} P_{p} P_{\sigma}$. Hence we make the 
transformation

$$
x_{i}=x_{1}^{\prime} y_{i}+x_{2}^{\prime} p_{i}+x_{3}^{\prime} \sigma_{i}
$$$$
(i=1,2,3) \text {, }
$$

where $x_{1}^{\prime}, x_{2}^{\prime}, x_{3}^{\prime}$ are proportional to the coordinates of a point referred to the triangle $P_{y} P_{\rho} P_{\sigma}$. Making use of (4) we find

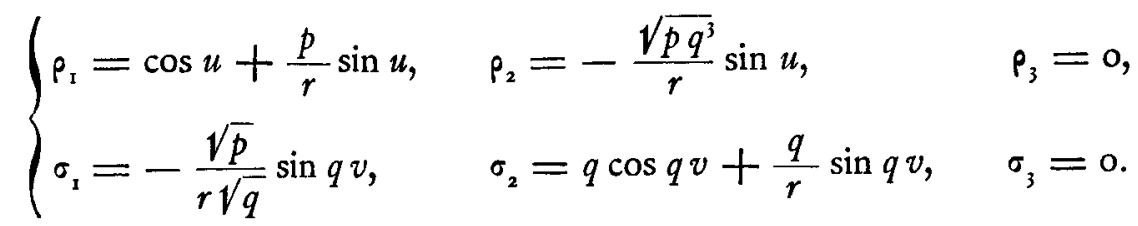

From (65), (66), (68) and (69) we obtain (after dropping accents)

$$
p q x_{2}^{2}+2 \sqrt{p q} \Delta_{u} x_{2} x_{3}+2 \sqrt{p q} \Delta x_{1} x_{3}+\left[\Delta^{2}+\Delta_{u}^{2}\right] x_{3}^{2}=0,
$$

$\Delta$ being defined by (62), as the equation of the curves $v=$ const. referred to the local triangle of reference $P_{y} P_{\rho} P_{\sigma}$. By means of the same transformation we find that the equation (67) of the curves $u=$ const. takes the form

$$
p q x_{3}^{2}+2 \sqrt{p q} \Delta_{v} x_{2} x_{3}+2 \sqrt{p q} \Delta x_{1} x_{2}+\left[\Delta^{2}+\Delta_{v}^{2}\right] x_{2}^{2}=0 .
$$

By assigning a fixed value to each of the arbitrary constants $p, q, r$ we may determine uniquely for $u=u_{o}, v=v_{o}$ the expressions $\Delta / \sqrt{p q}, \Delta_{u} / \sqrt{p q}$ and $\Delta_{v} / \sqrt{p q}$ occurring in the coefficients of $\left(66^{\prime}\right)$ and $\left(67^{\prime}\right)$ which is equivalent to choosing two arbitrary conics $u=u_{\mathrm{o}}$ and $v=v_{\mathrm{o}}$ as curves of the net.

The Laplacian transform is the line $P_{\rho} P_{\sigma}$.

Hence we may formulate the following theorem.

Choose an arbitrary triangle $L M N$. Trough the point $L$ draw two arbitrary conics $C$ and $C^{\prime}$ tangent to $L N$ and $L M$ respectively at $L$. There exists one and only one net of conics for which $H=K=\mathbb{E}=\mathbb{C}^{\prime \prime}=\mathrm{O}$ which contains $C$ and $C^{\prime}$ and which has for its degenerate first and minus first LAPLACIan transforms the line $M N$.

Chicago, Illinois, 14 July I915.

Jasper Ole Hassler. 